\title{
Critical number of atoms in an attractive Bose-Einstein condensate on an optical plus harmonic traps
}

\author{
Sadhan K. Adhikari \\ Instituto de Física Teórica, Universidade Estadual Paulista, 01.405-900 São Paulo, \\ São Paulo, Brazil
}

\begin{abstract}
.
The stability of an attractive Bose-Einstein condensate on a joint one-dimensional optical lattice and an axially-symmetric harmonic trap is studied using the numerical solution of the time-dependent mean-field Gross-Pitaevskii equation and the critical number of atoms for a stable condensate is calculated. We also calculate this critical number of atoms in a double-well potential which is always greater than that in an axially-symmetric harmonic trap. The critical number of atoms in an optical trap can be made smaller or larger than the corresponding number in the absence of the optical trap by moving a node of the optical lattice potential along the axial direction of the harmonic trap. This variation of the critical number of atoms can be observed experimentally and compared with the present calculation.
\end{abstract}

PACS numbers: 03.75.-b 
The successful detection [1,2] of a Bose-Einstein condensate (BEC) of dilute weaklyinteracting trapped bosonic atoms at ultra-low temperatures initiated intense theoretical activities on different aspects of the condensate. The experimental magnetic trap is usually axially symmetric. More recently in different experiments a periodic opticallattice potential generated by a standing-wave laser field has been employed along the axial direction of the magnetic trap $[3,4,4,6,6,6,8$. The optical-lattice potential has been used in the study of matter-wave interference [3], of oscillating atomic current in a one dimensional array of Josephson junctions [5], of Bloch oscillation and Landau Zenner tunneling [6] and of superfluid-insulator classical [7] and quantum [4] phase transitions among others. The periodic optical-lattice potential has also been useful in the generation of matter-wave bright soliton [8]. There are many theoretical studies on a BEC in a periodic optical-lattice potential [9].

Most of the above experiments were performed employing atoms with repulsive interaction, where the BEC is stable for any number of atoms. However, atoms with attractive interaction have been used in some experiments [2, 8, 10, 11, where the BEC is stable for the number of atoms smaller than a critical number [12, 13, 14. When the number of atoms increases beyond this critical value, due to interatomic attraction the radius of the BEC tends to zero and the central density of the condensate tends to infinity. Consequently, the condensate collapses while emitting atoms in an exploding fashion due to three-body recombination until the number of atoms is reduced below the critical number and a stable configuration is reached [2]. With a supply of atoms from an external source the condensate can grow again and a series of collapse and explosion can take place and has been observed in a BEC of ${ }^{7} \mathrm{Li}$ atoms [2. The attractive condensate has been fundamental in the generation of matter-wave bright soliton [8] and in the study of collapse and explosion of a condensate simulating the supernova explosion of a star [15].

The critical number of atoms in an attractive condensate in an axially-symmetric trap has been studied experimentally [2, 10, 11] and numerically [12, 13, 14] by various authors under different symmetries extending from a pancake shaped BEC through spherical to a cigar shaped one. When the axial trapping frequency $\omega_{z}$ equals the radial trapping frequency $\omega_{\rho}$ one has a spherical BEC. When $\omega_{z}>>\omega_{\rho}\left(<<\omega_{\rho}\right)$ one has a pancake (cigar) shaped BEC. In view of recent experiments $[\underline{3}, 4,4,5,6$, ,, ,, on a cigarshaped BEC trapped jointly in a axially-symmetric harmonic plus a optical-lattice trap, we investigate the stability of an attractive cigar-shaped condensate in such a trap using the direct numerical solution of the mean-field Gross-Pitaevskii (GP) equation [16] with appropriate trapping potential without any simplifying assumption.

Specifically, we calculate the critical number of atoms in an attractive BEC trapped jointly in an axial and optical-lattice traps. This will have direct consequence on the study of the generation and movement of bright solitons as in the recent experiment [8] with similar traps. We shall see that this critical number could be increased by appropriately setting the optical-lattice trap in the axial direction. A larger critical number is always welcome from the experimental consideration. Also, as the results 
of the present mean-field study could be verified experimentally, this will provide a stringent test of the applicability of the GP equation on the study of collapse of an attractive condensate in the presence of a joint optical plus axially-symmetric traps. The most recent experiment [1] on the critical number in an axially-symmetric trap alone agrees well with the theoretical predictions [13, 14.

There have been many theoretical studies [17] of a BEC in a double-well potential and an experimental study of the same seems to be under control [18. In view of this we also present a study of the critical number of atoms in an attractive condensate in a double-well potential trap.

The time-dependent BEC wave function $\Psi(\mathbf{r} ; \tau)$ at position $\mathbf{r}$ and time $\tau$ is described by the following mean-field nonlinear GP equation [16]

$$
\left[-i \hbar \frac{\partial}{\partial \tau}-\frac{\hbar^{2} \nabla^{2}}{2 m}+V(\mathbf{r})+g N|\Psi(\mathbf{r} ; \tau)|^{2}\right] \Psi(\mathbf{r} ; \tau)=0
$$

where $m$ is the mass and $N$ the number of atoms in the condensate, $g=4 \pi \hbar^{2} a / m$ the strength of interatomic interaction, with $a$ the atomic scattering length. In the presence of the combined axially-symmetric and optical-lattice traps $V(\mathbf{r})=\frac{1}{2} m \omega^{2}\left(\rho^{2}+\nu^{2} z^{2}\right)+$ $V_{\text {aux }}$ where $\omega$ is the angular frequency of the harmonic trap in the radial direction $\rho, \nu \omega$ that in the axial direction $z$, with $\nu$ the aspect ratio, and $V$ aux is an auxiliar potential simulating a double-well or optical-lattice trap introduced later. The normalization condition is $\int \mathrm{d} \mathbf{r}|\Psi(\mathbf{r} ; \tau)|^{2}=1$.

In the axially-symmetric configuration, the wave function can be written as $\Psi(\mathbf{r}, \tau)=\psi(\rho, z, \tau)$. Now transforming to dimensionless variables $x=\sqrt{2} \rho / l, y=$ $\sqrt{2} z / l, t=\tau \omega$, with the harmonic oscillator length $l \equiv \sqrt{\hbar /(m \omega)}$, and $\varphi(x, y ; t) \equiv$ $x \sqrt{l^{3} / \sqrt{8}} \psi(\rho, z ; \tau)$, (11) becomes [19]

$$
\begin{aligned}
& {\left[-i \frac{\partial}{\partial t}-\frac{\partial^{2}}{\partial x^{2}}+\frac{1}{x} \frac{\partial}{\partial x}-\frac{\partial^{2}}{\partial y^{2}}+\frac{1}{4}\left(x^{2}+\nu^{2} y^{2}\right)\right.} \\
& \left.+\frac{V_{\text {aux }}}{\hbar \omega}-\frac{1}{x^{2}}+8 \sqrt{2} \pi n\left|\frac{\varphi(x, y ; t)}{x}\right|^{2}\right] \varphi(x, y ; t)=0,
\end{aligned}
$$

where nonlinearity $n=N a / l$. In terms of the one-dimensional probability $P(y, t) \equiv 2 \pi$ $\int_{0}^{\infty} \mathrm{d} x|\varphi(x, y, t)|^{2} / x$, the normalization of the wave function is given by $\int_{-\infty}^{\infty} \mathrm{d} y P(y, t)=$ 1 .

A double-well potential for an axially-symmetric trap can be simulated by taking

$$
\frac{V_{\mathrm{aux}}}{\hbar \omega} \equiv \frac{V_{\mathrm{dw}}}{\hbar \omega}=V_{0} \exp \left(-y^{2}\right)
$$

where $V_{0}$ is the strength of the double-well potential $V_{\mathrm{dw}}$. The optical potential created with a standing-wave laser field of wavelength $\lambda$ along the axial direction can be represented by taking $V_{\text {aux }} \equiv V_{\text {opt }}=V_{0} E_{R} \cos ^{2}\left(k_{L} z\right)$, with $E_{R}=\hbar^{2} k_{L}^{2} /(2 m)$, $k_{L}=2 \pi / \lambda$, and $V_{0}$ the strength [3, 4, 5, 6]. This periodic potential has a maximum at the center of the harmonic trap $z=0$. The center of the harmonic trap could also be made to coincide with a minimum of the periodic potential or anywhere in between. All these cases can be covered by taking a phase $\delta$ in the $\cos ^{2}\left(k_{L} z\right)$ term 


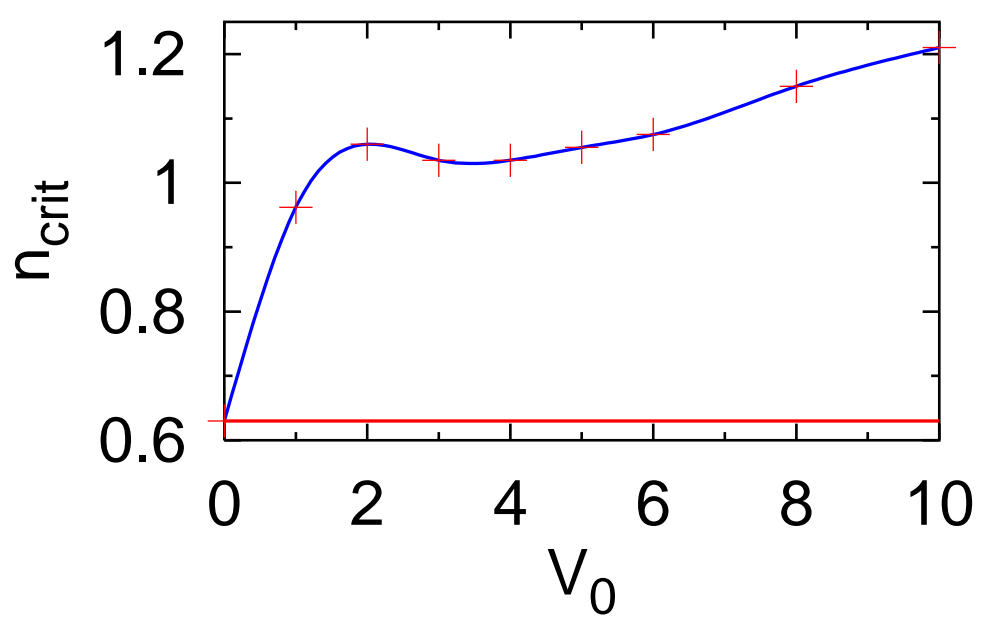

Figure 1. The reduced critical number $n_{\text {crit }}$ vs. strength $V_{0}$ of the double-well potential for $\nu=0.5$ : line with $+\rightarrow$ double well; straight line $n_{\text {crit }}=0.63 \rightarrow V_{0}=0$.

so that $V_{\text {opt }}=V_{0} E_{R} \cos ^{2}\left(k_{L} z+\delta\right)$. In terms of the dimensionless laser wave length $\lambda_{0}=\sqrt{2} \lambda / l$ and a dimensionless standing-wave energy parameter $E_{R} /(\hbar \omega)=4 \pi^{2} / \lambda_{0}^{2}$, the periodic optical-lattice potential $V_{\text {opt }}$ of (2) is

$$
\frac{V_{\mathrm{aux}}}{\hbar \omega} \equiv \frac{V_{\mathrm{opt}}}{\hbar \omega}=V_{0} \frac{4 \pi^{2}}{\lambda_{0}^{2}}\left[\cos ^{2}\left(\frac{2 \pi}{\lambda_{0}} y+\delta\right)\right] .
$$

We shall see that by appropriately choosing the value of $\delta$ and $\lambda_{0}$ the critical number of atoms in an attractive BEC could be increased after the introduction of the optical trap.

We solve (2) numerically using a split-step time-iteration method with the CrankNicholson discretization scheme described recently [19]. The time iteration is started with the harmonic oscillator solution of (2) with $n=0: \varphi(x, y)=\left[\nu /\left(8 \pi^{3}\right)\right]^{1 / 4}$ $x e^{-\left(x^{2}+\nu y^{2}\right) / 4}\left[19\right.$. The nonlinearity $n$ and the optical-lattice potential parameter $V_{0}$ are slowly changed by equal amounts in $5000 \mathrm{n}$ steps of time iteration until the desired value of $n$ and $V_{0}$ are attained for the double-well or the optical-lattice potential. Then, without changing any parameter, the solution so obtained is iterated 5000 times so that a stable solution is obtained independent of the initial input and time and space steps.

For a repulsive condensate the scattering length $a$ is positive. For an attractive condensate the scattering length $a$ and the nonlinearity $n$ are negative. In the calculation we find that, for an attractive condensate with negative $n$, stable solution of the GP equation cannot be obtained for $|n|$ greater than a critical value $n_{\text {crit }}$ - the reduced critical number. For the spherically symmetric case $n_{\text {crit }}=0.5746[13$. The critical value for the number of atoms is given by $N_{\text {crit }}=n_{\text {crit }} l /|a|$. The dimensionless parameter $n_{\text {crit }}$ is universal, whereas the number $N_{\text {crit }}$ depends on the typical experimental set up, e.g., the atomic scattering length and the harmonic oscillator length of the trap. 

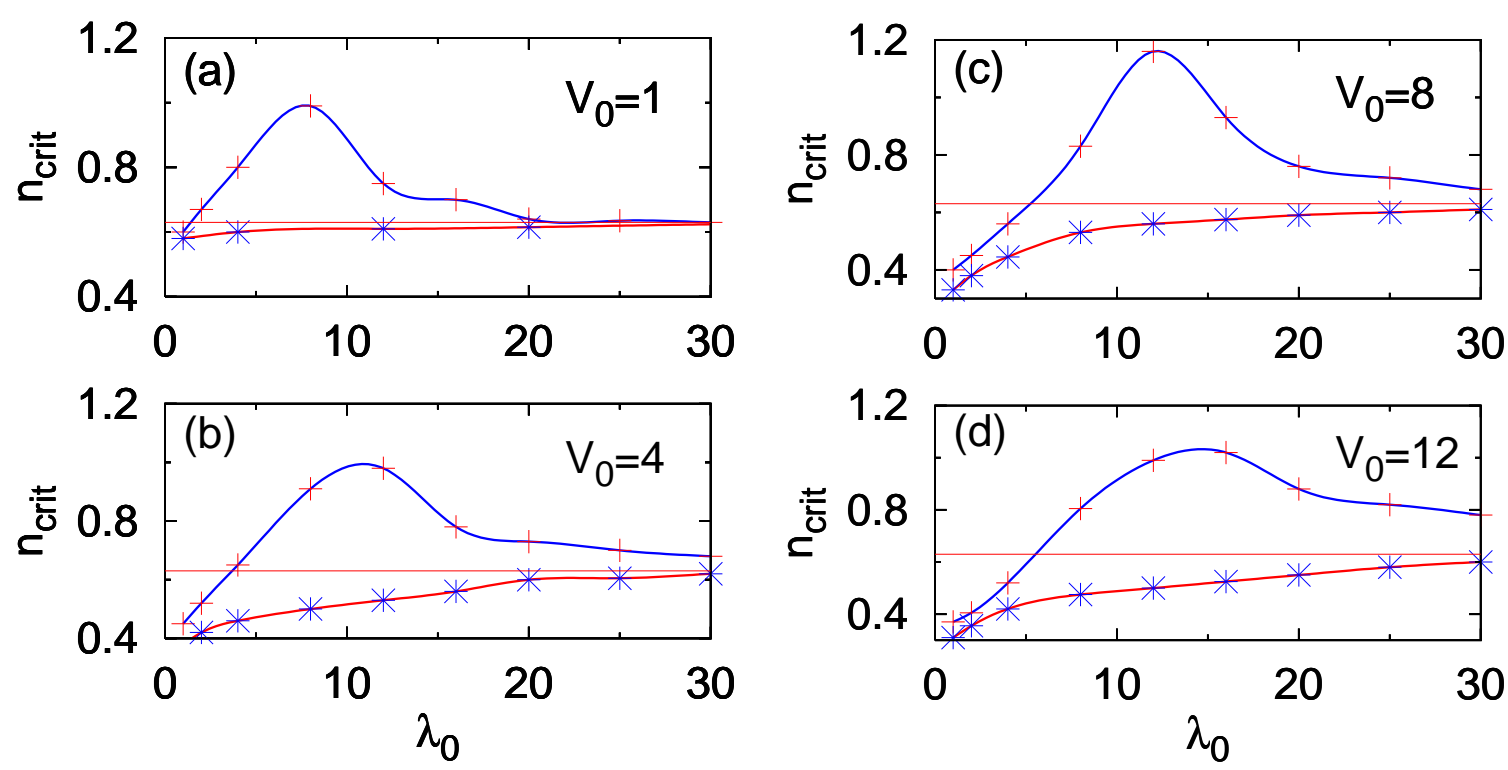

Figure 2. The reduced critical number $n_{\text {crit }}$ vs. dimensionless wave length $\lambda_{0}$ for $\nu=0.5$ and (a) $V_{0}=1$, (b) $V_{0}=4$, (c) $V_{0}=8$, and (d) $V_{0}=12$ : line with $+\rightarrow \delta=0$; line with $\star \rightarrow \delta=\pi / 2$; straight line $n_{\text {crit }}=0.63 \rightarrow V_{0}=0$.

First we present a study of the critical number $n_{\text {crit }}$ for the double-well potential. We consider an elongated cigar-shaped harmonic trap with $\nu=0.5$ although the calculation can be extended for other values of $\nu$. In figure 1 we plot $n_{\text {crit }}$ vs. $V_{0}$. The case $V_{0}=0$ corresponds to the absence of the optical-potential or double-well trap and in this case we obtain $n_{\text {crit }}=0.63$ in agreement with $k_{\text {crit }} \equiv n_{\text {crit }} \nu^{1 / 6}=0.56$ reported in reference [13] for $\nu=0.5$. We find from figure 1 that $n_{\text {crit }}$ for the doublewell potential is always larger than that for the corresponding harmonic axial trap. The double-well trap separates the original condensate effectively into two parts with a region of low density in between. When $V_{0}$ is large this separation is more pronounced and one effectively has two separated condensates and the critical number of atoms in the double-well trap tends approximately to twice the critical number in the corresponding harmonic trap alone. For $V_{0}=0$ the double-well is absent and $n_{\text {crit }}=0.63$. Figure 1 shows the evolution of $n_{\text {crit }}$ between these two limiting values. It should be recalled that the exact limiting value for $n_{\text {crit }}$ for large $V_{0}$ as well as the evolution between the two limits will depend on the type of the double-well potential employed. Figure 1 represents these general features for the double-well potential (3).

In most experimental set ups with an optical-lattice potential a cigar shaped trap has been used and we consider only this symmetry $(\nu<1)$ in this paper. Here we consider the anisotropy parameter $\nu=0.5$ and four values of the strength $V_{0}$ of the optical-lattice potential: $V_{0}=1,4,8$ and 12. We consider the two limiting cases $\delta=0$ and $\pi / 2$ in the optical-lattice periodic potential (4).

The critical nonlinearity $n_{\text {crit }}$ vs. dimensionless wave length $\lambda_{0}$ for different $V_{0}$ and $\delta(=0, \pi / 2)$ are plotted in figures 2. This constant critical value $\left(n_{\text {crit }}=0.63\right)$ 

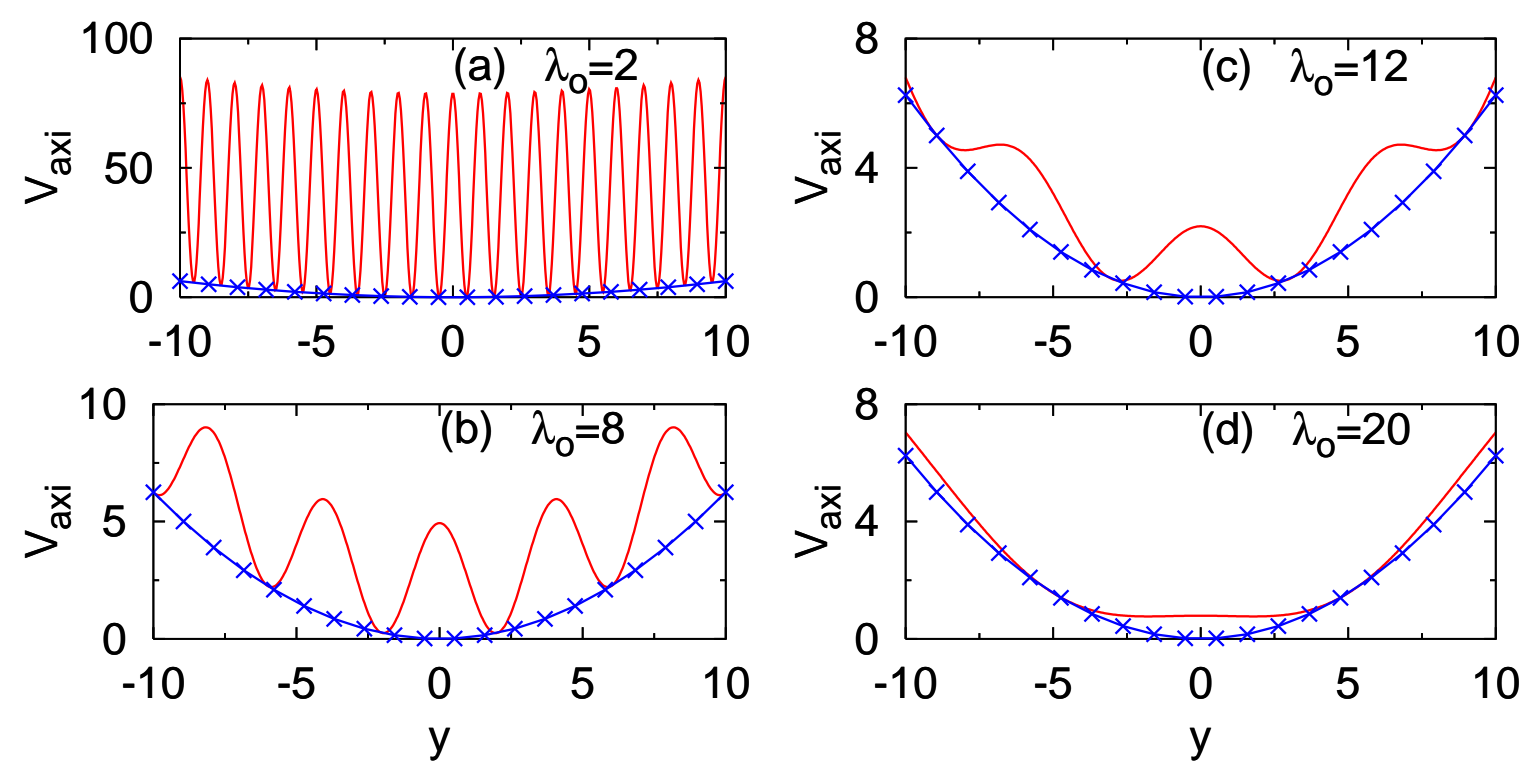

Figure 3. The full axial potential (55) $V_{\text {axi }}$ vs. $y$ for $V_{0}=8, \delta=0$ and $\nu=0.5$ for (a) $\lambda_{0}=2$, (b) $\lambda_{0}=8$, (c) $\lambda_{0}=12$, and (d) $\lambda_{0}=20$ : full line $\rightarrow V_{\text {axi }}$; full line with $\times \rightarrow$ axial harmonic potential $\nu^{2} y^{2} / 4$.

for $\nu=0.5$ and $V_{0}=0$ is also shown in figures 2 for a comparison. The lines with + represent $\delta=0$ or the $\cos ^{2}\left(2 \pi y / \lambda_{0}\right)$ dependence of $V_{\text {opt }}$ whereas the lines with $\star$ represent $\delta=\pi / 2$ or the $\sin ^{2}\left(2 \pi y / \lambda_{0}\right)$ dependence. For a fixed $V_{0}$ and $\delta=0$ one has $n_{\text {crit }}<0.63$ for small $\lambda_{0}$. With the increase of $\lambda_{0}, n_{\text {crit }}$ increases past 0.63 and eventually it decreases to 0.63 asymptotically for a large enough $\lambda_{0}$. For $\delta=\pi / 2$ and small $\lambda_{0}, n_{\text {crit }}$ starts at a value very similar to the $\delta=0$ case. With the increase of $\lambda_{0}, n_{\text {crit }}$ monotonically increases in this case to the value 0.63 , asymptotically, however, remaining always smaller than 0.63 . The plots of $n_{\text {crit }}$ vs. $\lambda_{0}$ for any $\delta$ lie between the $\delta=0$ and $\delta=\pi / 2$ limits shown in figures 2 .

The behavior of the $n_{\text {crit }}$ vs. $\lambda_{0}$ curves reported in figures 2 can be understood from a consideration of the total axial potential

$$
V_{\text {axi }}=\frac{1}{4} \nu^{2} y^{2}+V_{0} \frac{4 \pi^{2}}{\lambda_{0}^{2}}\left[\cos ^{2}\left(\frac{2 \pi}{\lambda_{0}} y+\delta\right)\right] .
$$

It should be noted that in the presence of the periodic optical-lattice potential the radial trap remains unchanged as the optical lattice does not provide any confinement in the radial direction. In figures 3 we plot $V_{\text {axi }}$ vs. $y$ for four different values of wavelength $\lambda_{0}$ for $V_{0}=8$ and $\delta=0$. For $\lambda_{0}=2$ we have a large number of nodes in the potential $V_{\text {axi }}$ revealing a large number of confining traps of the periodic potential as one can find in figure 3 (a). The number of available traps of $V_{\text {axi }}$ in a certain region of space in the axial $y$ direction decreases as $\lambda_{0}$ increases (to 12 through 8 ) as found in figures 3 (b) and (c). Eventually, for a large enough $\lambda_{0}(\sim 20)$ the confining traps of the periodic potential $V_{\text {axi }}$ disappear and it reduces essentially to the harmonic trap potential as in figure 3 (d). For large $\lambda_{0}$ the extention of each of the periodic traps of $V_{\text {axi }}$ is larger 

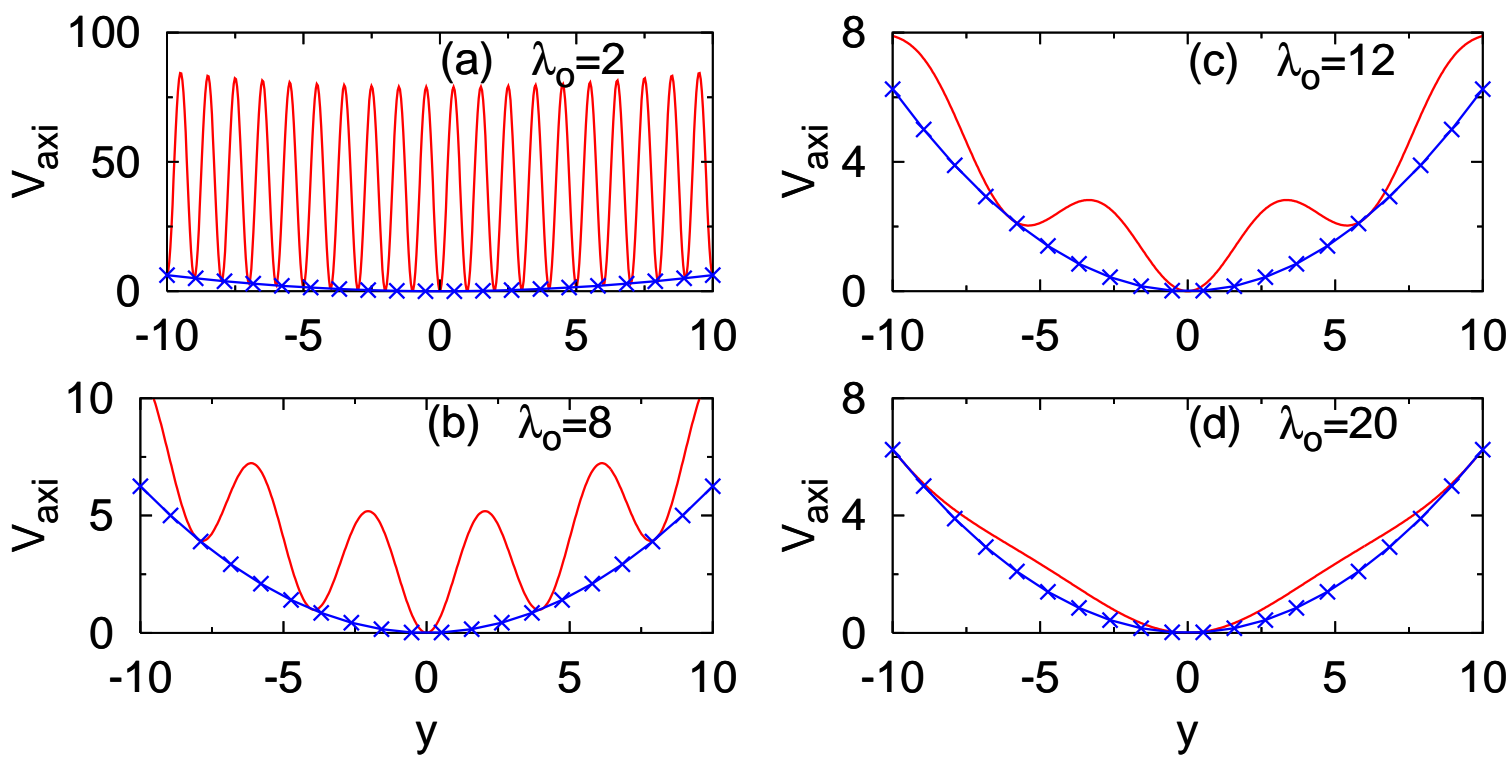

Figure 4. Same as in figures 3 but for $\delta=\pi / 2$

than that of the axial harmonic trap, and $V_{\text {axi }}$ tends towards the axial harmonic trap $\nu^{2} y^{2} / 4$. Hence one can safely conclude that for large $\lambda_{0}, n_{\text {crit }}$ tends to its value $(=0.63)$ in the absence of the optical potential as one can find from figures 2 .

The typical axial dimension of the attractive condensate before collapse is larger than the width of a single confining trap of the optical potential for small $\lambda_{0}$ (figure 3 (a)), hence the condensate should occupy a few of these confining optical-lattice traps. Consequently, the BEC will be squezeed towards the center of each these confining traps with a region of low density between the traps. Because of this further squeezing the BEC will collapse with a smaller number of atoms and lead to a $n_{\text {crit }}$ smaller than that in the absence of the optical trap. This effect will be large for a stronger optical potential with large $V_{0}$ as one can see in figures 2 . The typical dimension of the attractive condensate in the axial direction is smaller or comparable to the dimension of a single trap of the optical potential for larger $\lambda_{0}$. For $\delta=0$ the appearance of a maximum of the axial potential $V_{\text {axi }}$ at $y=0$ pushes the condensate apart into two nearby minima of the axial potential. Consequently, in these cases the BEC swells and one effectively has two separated pieces of the condensate in two traps. In this case the central part of the total trap, where the BEC is formed, is similar to a double-well potential. Consequently, there is more space available for the full condensate and hence $n_{\text {crit }}$ can grow beyond the critical value in the absence of the optical trap.

In figures 4 we plot $V_{\text {axi }}$ vs. $y$ for four different values of wavelength $\lambda_{0}$ for $V_{0}=8$ and $\delta=\pi / 2$. In this case one has a minimum of the axial potential $V_{\text {axi }}$ at $y=0$ in contrast to the $\delta=0$ case where one has a maximum of $V_{\text {axi }}$ at $y=0$. Consequently, for larger $\lambda_{0}$ the BEC will be essentially squeezed into the central trap of the optical-lattice potential. The central part of the total potential, where the BEC is formed, is never of 
the double-well type, but rather like a stronger single-well type. Because of the above squeezing the critical number of atoms will always be less than the critical number in the absence of the optical-lattice trap. Again $n_{\text {crit }}$ grows monotonically between the small and large $\lambda_{0}$ limits as one can see in figures 2 .

In conclusion, using the numerical solution of the GP equation we have studied the stability of an attractive BEC trapped jointly by an optical and an axially-symmetric harmonic traps. We calculate the critical number of atoms for stability in this case. We find that the reduced critical number $n_{\text {crit }}=N_{\text {crit }}|a| / l$ could either increase or decrease in relation to the same number in the absence of the optical trap. However, for most experimental set ups $\lambda_{0}$ is expected to be less than 3 and and from figures 2 we find that $n_{\text {crit }}$ should reduce after the introduction of the optical lattice trap. For a double-well potential $n_{\text {crit }}$ is always larger than the same for the corresponding axiallysymmetric harmonic trap. A prior knowledge of the critical number $n_{\text {crit }}$ as obtained in this paper will be useful in the experimental generation and study of matter-wave bright solitons under similar trapping conditions. Also, $n_{\text {crit }}$ can be measured experimentally and compared with the present theoretical results. This will be helpful in evaluating the applicability of the mean-field GP equation in the study of attractive condensates trapped in a joint periodic optical plus a harmonic potential.

\section{Acknowledgments}

The work was supported in part by the CNPq and FAPESP of Brazil.

\section{Reference}

[1] Anderson M H, Ensher J R, Matthews M R, Wieman C E and Cornell E A 1995 Science 269198 Ensher J R, Jin D S, Matthews M R, Wieman C E and Cornell E A 1996 Phys. Rev. Lett. 77 4984

Davis K B, Mewes M O, Andrews M R, van Druten N J, Durfee D S, Kurn D M and Ketterle W 1995 Phys. Rev. Lett. 753969

Fried D G, Killian T C, Willmann L, Landhuis D, Moss S C, Kleppner D and Greytak T J 1998 Phys. Rev. Lett. 813811

Pereira Dos Santos F, Léonard J, Junmin Wang, Barrelet C J, Perales F, Rasel E, Unnikrishnan C S, Leduc M and Cohen-Tannoudji C 2001 Phys. Rev. Lett. 863459

[2] Bradley C C, Sackett C A, Tolett J Jand and Hulet R G 1995 Phys. Rev. Lett. 751687 Gerton J M, Strekalov D, Prodan I and Hulet R G 2000 Nature London 408692

[3] Anderson B P and Kasevich M A 1998 Science 2821686 Orzel C, Tuchman A K, Fenselau M L, Yasuda M and Kasevich M 2001 Science 2912386

[4] Greiner M, Mandel O, Esslinger T, Hänsch T W and Bloch I 2002 Nature (London) 41539 Greiner M, Mandel O, Hänsch T W and Bloch I 2002 Nature (London) 41951

[5] Cataliotti F S, Burger S, Fort C, Maddaloni P, Minardi F, Trombettoni A, Smerzi A and Inguscio M 2001 Science 293843

[6] Morsch O, Müller J H, Cristiani M, Ciampini D and Arimondo E 2001 Phys. Rev. Lett. 87140402 Morsch O, Cristiani M, Müller J H, Ciampini D and Arimondo E 2002 Phys. Rev. A 66021601

[7] Cataliotti F S, Fallani L, Ferlaino F, Fort C, Maddaloni P, Inguscio M, Smerzi A, Trombettoni A, Kevrekidis P G and Bishop A R 2002 e-print cond-mat/0207139 
[8] Strecker K E, Partridge G B, Truscott A G and Hulet R G 2002 Nature (London) 417150

[9] Band Y B and Trippenbach M 2002 Phys. Rev. A 65053602

Liu W M, Fan W B, Zheng W M, Liang J Q and Chui S T 2002 Phys. Rev. Lett. 88170408

Kramer M, Pitaevskii L and Stringari S 2002 Phys. Rev. Lett. 88180404

Goral K, Santos L and Lewenstein M 2002 Phys. Rev. Lett. 88170406

Sukhorukov A A and Kivshar Y S 2002 Phys. Rev. E 65036609

Adhikari S K 2003 Phys. Lett. A 308302

Adhikari S K 2003 J. Phys. B: At. Mol. Opt. Phys. 36 in press

Adhikari S K and Muruganandam P 2003 Phys. Lett. A 310229

[10] Roberts J L, Claussen N R, Cornish S L, Donley E A, Cornell E A and Wieman C E 2001 Phys. Rev. Lett. 864211

[11] Claussen N R, Kokkelmans S J J M F, Thompson S T, Donley E A and Wieman C E 2003 Preprint cond-mat/0302195

[12] Ruprecht P A, Holland M J, Burnett K and Edwards M 1995 Phys. Rev. A 514704

Houbiers M and Stoof H T C 1996 Phys. Rev. A 545055

Bergé L, Alexander T J and Kivshar Y S 2000 Phys. Rev. A 62023607

Huepe C, Métens S, Dewel G, Borckmans P and Brachet M E 1999 Phys. Rev. Lett. 821616

Huepe C, Tuckerman L S, Métens S, Brachet M E Preprint cond-mat/0212069

[13] Gammal A, Frederico T and Tomio L 2001 Phys. Rev. A 64055602

[14] Adhikari S K 2002 Phys. Rev. E 65016703

[15] Donley E A, Claussen N R, Cornish S L, Roberts J L, Cornell E A and Wieman C E 2001 Nature (London) 412295

[16] Dalfovo F, Giorgini S, Pitaevskii L P and Stringari S 1999 Rev. Mod. Phys. 71463

[17] Sakellari E, Leadbeater M, Kylstra N J and Adams C S 2002 Phys. Rev. A 66033612

Anglin J 1997 Phys. Rev. Lett. 796

Smerzi A, Fantoni S, Giovanazzi and Shenoy S R 1997 Phys. Rev. Lett. 794950

S, Giovanazzi, Smerzi A and Fantoni S 2000 Phys. Rev. Lett. 844521

D'Agosta R and Presilla C 2002 Phys. Rev. A 65043609

Mahmud K W, Kutz J N and Reinhardt W P 2002 Phys. Rev. A 66063607

[18] Tiecke T G, Kemmann M, Buggle C, Shvarchuck I, von Klitzing W and Walraven J T M $2003 \mathrm{~J}$. Opt. B: Quantum Semiclass. Opt. 5 S119

[19] Adhikari S K and Muruganandam P 2002 J. Phys. B: At. Mol. Opt. 352831 